\title{
Green the Power Grid with Stochastic Network Calculus
}

\author{
Kai Wang \\ Computer Science and Technology, Tsinghua University, P.R. China \\ Computing and Mathematical Sciences, California Institute of Technology, USA \\ kaiphd@ccaltech,edu
}

\begin{abstract}
The renewable energy generation will constitute an important part of the next generation grid. As the variations of renewable sources may not match the time distribution of load, energy storage is essential for grid stability. Supplemented with energy storage, we investigate the feasibility of integrating solar photovoltaic (PV) panels and wind turbines into the grid. To deal with the fluctuation in both the power generation and demand, we borrow ideas from stochastic network calculus and build a a stochastic model for the power supply reliability with different renewable energy congurations. To illustrate the validity of the model, we conduct a case study for the integration of renewable energy sources into the power system of an island off the coast of Southern California.
\end{abstract}

\section{PROBLEM STATEMENT}

We consider a scenario where the electric network is supplied by a combination of solar PV panels, wind turbines and battery, which work together to satisfy the energy demand. When the energy sources are abundant, the excess generation will feed the battery until it's fully charged. Whenever there is a defficiency in power, the battery will be discharged to cover the load requirements until the energy storage is depleted. We let $b(t)$ to denote the state of battery storage at time $t$ (in MWh).

In the face of fluctuations in both power load and generation, we try to investigate the effects of battery storage on the power supply reliability in congurations with different levels of renewable generation. We are interested in quantifying the Loss of Power Supply Probability (LPSP) at a given time, and the long-term expected Fraction of Time energy Not-Served (FTNS) during a time period. In general, the LPSP/FTNS is a function

Permission to make digital or hard copies of all or part of this work for personal or classroom use is granted without fee provided that copies are not made or distributed for profit or commercial advantage and that copies bear this notice and the full citation on the first page. To copy otherwise, to republish, to post on servers or to redistribute to lists, requires prior specific permission and/or a fee.

ACM CoNEXT 2011, December 6-9 2011, Tokyo, Japan.

Copyright 2011 ACM 978-1-4503-1042-0/11/0012 ...\$10.00. of the total area $N_{p}$ of PV panels, the number $N_{w}$ of wind turbines, and the capacity $C$ of battery storage.

\section{POWER SYSTEM MODELING}

The stochastic nature of the load and renewable generation motivates us to build a system model based on similar concepts in stochastic network calculus [1]. However, the concepts of stochastic network calculus could not be directly applied to the power network, we have to substantially adjust and modify the theory framework so as to analyze the power system.

Consider a power system where the time axis $T$ is discretized into 1 hour. In time interval $(0, t](t \leq T)$, we denote by $D(t)$ the cumulative amount of energy demand in the system (in MWh); by $S(t)$ the cumulative amount of energy supply in the system. We call $D(t)$ the energy demand process, and $\mathrm{S}(\mathrm{t})$ the energy supply process of the system, with $D(0)=S(0)=0$. For any $0 \leq s \leq t$, we denote $D(s, t)=D(t)-D(s)$, and $S(s, t)=S(t)-S(s)$. In this paper, we use $[x]^{+}$to denote the maximum of a given number of $x$ and 0 .

The energy demand curve implies a upper bound of the cumulative energy demand. By introducing a violation probability, we can find its stochastic counterpart. Throughout this paper, we assume all demand curve, supply curve functions are non-negative and nondecreasing, and all bounding functions are non-negative and non-increasing. The proofs for the Lemmas and Theorem below are omitted due to page limitations.

Definition 1. A energy demand process $D(t)$ is said to have a stochastic demand curve $\alpha(t)$ with bounding function $\varepsilon_{d}$, denoted by $D \sim\left\langle\varepsilon_{d}, \alpha\right\rangle$, if for all $t \geq 0$, and all $x \geq 0$, there holds $\mathbb{P}\left\{\sup _{0 \leq s \leq t}[D(s, t)-\alpha(t-s)]>\right.$ $x\} \leq \varepsilon_{d}(x)$.

The energy supply curve defines a lower bound on the energy provided by the power generator. By introducing a violation probability, we can make sure that the power supply process is above some lower bound with a high probability.

Definition 2. A energy supply process $S(t)$ is said to provide a stochastic supply curve $\beta(t)$ with bounding 
function $\varepsilon_{s}$, denoted by $S \sim\left\langle\varepsilon_{s}, \beta\right\rangle$, if for all $t \geq 0, x \geq$ 0 , there holds $\mathbb{P}\left\{\sup _{0 \leq s \leq t}[\beta(t-s)-S(s, t)]>x\right\} \leq \varepsilon_{s}(x)$.

We then analogize the energy demand and supply to a virtual queueing system. Regarding the power demand as the arrival of the queue, the energy supply as the service provided by the queue, then the buffering process of the virtual queue could be considered as the discharging process of the battery. We denote the buffer for the virtual queueing system at time $t$ as $B(t)$, which can be considered as the deficit energy storage of the system, i.e., $B(t)=C-b(t)$.

When the power generated is greater than the power demand at a certain period of time, as long as the maximum battery capacity is not reached, the buffer decreases which means that the battery is charged. When the load is greater than the power supply in a time period, the buffer increases, i.e., the battery is discharged until it decreases to the minimum level. We have:

$B(t)=\min \left[C(t),[B(t-1)+D(t-1, t)-S(t-1, t)]^{+}\right]$.

For a given battery storage capacity $C$, we are interested in the probability that the energy demand cannot be met by the generation and the stored energy in the battery. Without loss of generality, the battery is fully charged initially, i.e., $B(0)=0$. We also assume that the energy demand that is not satisfied in a time slot would be defferred to the next one. Then we can derive a non-recursive identity for the virtual buffer size:

$$
B(t)=\sup _{0 \leq s \leq t}[D(s, t)-S(s, t)] .
$$

In time slot $t(t \geq 1)$, if $[B(t-1)+D(t-1, t)-$ $S(t-1, t)]^{+}>C$, we call this loss of power supply at time $t$, which is dependent on the storage capacity $C$ (in MWh). The Loss of Power Supply satisfies $L P S(t)=$ $[B(t-1)+D(t-1, t)-S(t-1, t)-C]^{+}$. With the battery storage capacity $C$, the $\operatorname{LPSP}(t)$ at time $t$ equals to $\mathbb{P}\{L P S(t)>0\}$. The FTNS(T), is given by:

$$
F T N S(T)=\frac{1}{T} \Sigma_{t=1}^{T} L P S P(t) .
$$

We introduce the following Lemma for LPSP:

THEOREM 1. For a virtual queue in the power network, if the energy demand process has a stochastic demand curve $\alpha$ with bounding function $\varepsilon_{d}$, i.e., $D \sim$ $\left\langle\varepsilon_{d}, \alpha\right\rangle$, and the system provides a stochastic supply curve $\beta$ with bounding function $\varepsilon_{s}$, i.e., $S \sim\left\langle\varepsilon_{s}, \beta\right\rangle$, then for all $t \geq 0, x \geq 0$, given storage capacity $C, \operatorname{LPSP}(t)$ can be calculated by:

$$
\operatorname{LPSP}(t) \leq \varepsilon_{d} \otimes \varepsilon_{s}(C-\alpha \oslash \beta(t))[1],
$$

where the operator $\otimes$ denotes the min-plus Convolution, and $\oslash$ denotes the min-plus De-convolution.

\section{PERFORMANCE EVALUATIONS}

We consider the Santa Catalina Island, which is 26 miles off the coast of CA, and investigate the feasibility
Table 1: FTNS vs. Battery Storage Capacity

\begin{tabular}{|l|c|c|c|c|}
\hline PV Units $\left(\times 10^{3}\right)$ & 2 & 3 & 4 & 5 \\
\hline Battery Capacity (MWh) & 51.7 & 38.2 & 34.6 & 33.2 \\
\hline
\end{tabular}

of replacing diesel generation with renewable resources. We use hourly data profiles of 31 days in Jan, 2010 for analytical study, which are obtained at locations near the Island with similar meteorological characteristics. The energy generation profile is based on the TMY data sets derived from the NSRDB archives [2], the per unit PV generation data for Long Beach, CA, is calculated by SAM [3]. The data for a wind turbine located off an island near Santa Barbara is obtained from the NREL [4]. The load profiles are obtained by personal communication with researchers from SCE [5].

With the data given above, using curve fitting, we can get the bounding function with respect to the stochastic demand/supply curves according to Definitions 1 and 2 . The demand/supply curves is based on the long time mean rate, and we use some convex exponential decay functions to fit the bounding functions. To aggregate heterogeneous power supply sources together, we have:

Theorem 2. (Aggregation Property) Consider a system that consists $N$ energy generators in parallel. If each power generator $(n=1,2, \ldots, N)$ provides a stochastic energy supply curve $S_{n} \sim\left\langle\varepsilon_{n}, \beta_{n}\right\rangle$, then the power system provides a stochastic supply curve $S \sim\langle\varepsilon, \beta\rangle$ with $\beta(t)=\beta_{1}(t)+\ldots+\beta_{N}(t), \varepsilon(x)=\varepsilon_{1} \otimes \ldots \otimes \varepsilon_{N}(x)$.

With difierent configurations of power generation, the aggregate energy supply from the solar and wind power can be calculated based on Lemma 2 . For a given battery capacity $C$, the $L P S P$ is provided by Lemma 1 . Together with (1), we can get the FTNS over the time period of the given data profile. For a targeting FTNS value of 0.01 , Table 1 shows the FTNS versus battery capacity for different levels of solar generation with a single wind turbine. We notice that FTNS decreases as the battery capacity increases. As $N_{p}$ increases, the FTNS decreases with the same battery capacity. We also notice that given a target $F T N S$ value, further increasing the $P V$ panels would have less impact in reducing the battery requirement due to the fluctuating nature of the power sources.

\section{ACKNOWLEDGMENT}

The research is supported by the China State Scholarship Fund, and the NSFC (No. 61020106002/60973107).

\section{REFERENCES}

[1] YM Jiang, Y Liu. Stochastic Network Calculus. Springer, 2008.

[2] http://rredc.nrel.gov/solar/old_data/nsrdb/

[3] https://www.nrel.gov/analysis/sam/

[4] http://wind.nrel.gov/Web nrel/

[5] http://www.sce.com/AboutSCE/Regulatory 